\title{
A Particular Solution of a Painlevé System in Terms of the Hypergeometric Function ${ }_{n+1} F_{n}{ }^{\star}$
}

Takao SUZUKI

Department of Mathematics, Kobe University, Rokko, Kobe 657-8501, Japan

E-mail:suzukit@math.kobe-u.ac.jp

Received June 23, 2010, in final form September 29, 2010; Published online October 07, 2010

doi:10.3842/SIGMA.2010.078

\begin{abstract}
In a recent work, we proposed the coupled Painlevé VI system with $A_{2 n+1^{-}}^{(1)}$ symmetry, which is a higher order generalization of the sixth Painlevé equation $\left(P_{\mathrm{VI}}\right)$. In this article, we present its particular solution expressed in terms of the hypergeometric function ${ }_{n+1} F_{n}$. We also discuss a degeneration structure of the Painlevé system derived from the confluence of ${ }_{n+1} F_{n}$.
\end{abstract}

Key words: affine Weyl group; generalized hypergeometric functions; Painlevé equations

2010 Mathematics Subject Classification: 17B80; 33C20; 34M55

\section{Introduction}

The main object in this article is the coupled Painlevé VI system with $A_{2 n+1}^{(1)}$-symmetry given in $[1,4]$, or equivalently, the Hamiltonian system $\mathcal{H}_{n+1,1}$ given in [6]. It is expressed as a Hamiltonian system on $\mathbb{P}^{1}(\mathbb{C})$

$$
t(t-1) \frac{d q_{i}}{d t}=\frac{\partial H}{\partial p_{i}}, \quad t(t-1) \frac{d p_{i}}{d t}=-\frac{\partial H}{\partial q_{i}}, \quad i=1, \ldots, n
$$

with

$$
\begin{aligned}
H= & \sum_{i=1}^{n} H_{\mathrm{VI}}\left[\sum_{j=0}^{n} \alpha_{2 j+1}-\alpha_{2 i-1}-\eta, \sum_{j=0}^{i-1} \alpha_{2 j}, \sum_{j=i}^{n} \alpha_{2 j}, \alpha_{2 i-1} \eta ; q_{i}, p_{i}\right] \\
& +\sum_{1 \leq i<j \leq n}\left(q_{i}-1\right)\left(q_{j}-t\right)\left\{\left(q_{i} p_{i}+\alpha_{2 i-1}\right) p_{j}+p_{i}\left(q_{j} p_{j}+\alpha_{2 j-1}\right)\right\},
\end{aligned}
$$

where $H_{\mathrm{VI}}$ is the Hamiltonian for $P_{\mathrm{VI}}$ defined as

$$
\begin{aligned}
H_{\mathrm{VI}}\left[\kappa_{0}, \kappa_{1}, \kappa_{t}, \kappa ; q, p\right]= & q(q-1)(q-t) p^{2}-\kappa_{0}(q-1)(q-t) p \\
& -\kappa_{1} q(q-t) p-\left(\kappa_{t}-1\right) q(q-1) p+\kappa q .
\end{aligned}
$$

Here $\alpha_{0}, \ldots, \alpha_{2 n+1}$ and $\eta$ are fixed parameters satisfying a relation $\sum_{i=0}^{2 n+1} \alpha_{i}=1$. We assume that the indices of $\alpha_{i}$ are congruent modulo $2 n+2$. Note that the system (1) includes $P_{\mathrm{VI}}$ as the case $n=1$. The aim of this article is to present a particular solution of the system (1) expressed in terms of the hypergeometric function ${ }_{n+1} F_{n}$.

${ }^{\star}$ This paper is a contribution to the Special Issue "Relationship of Orthogonal Polynomials and Special Functions with Quantum Groups and Integrable Systems". The full collection is available at http://www.emis.de/journals/SIGMA/OPSF.html 
The hypergeometric function ${ }_{n+1} F_{n}$ is defined by the power series

$$
{ }_{n+1} F_{n}\left[\begin{array}{c}
a_{0}, \ldots, a_{n} \\
b_{1}, \ldots, b_{n}
\end{array} ; t\right]=\sum_{i=0}^{\infty} \frac{\left(a_{0}\right)_{i}\left(a_{1}\right)_{i} \cdots\left(a_{n}\right)_{i}}{(1)_{i}\left(b_{1}\right)_{i} \cdots\left(b_{n}\right)_{i}} t^{i},
$$

where $(a)_{i}$ stands for the factorial function

$$
(a)_{i}=a(a+1) \cdots(a+i-1)=\frac{\Gamma(a+i)}{\Gamma(a)} .
$$

Denoting by $\delta=t d / d t$, we see that $x={ }_{n+1} F_{n}$ satisfies an $(n+1)$-th order linear differential equation

$$
\left[\delta\left(\delta+b_{1}-1\right) \cdots\left(\delta+b_{n}-1\right)-t\left(\delta+a_{0}\right) \cdots\left(\delta+a_{n}\right)\right] x=0,
$$

which is called the generalized hypergeometric equation [3]. The equation (2) is of Fuchsian type with regular singular points at $t=0,1, \infty$ and the Riemann scheme

$$
\left[\begin{array}{ccc}
t=0 & t=1 & t=\infty \\
0 & 0 & a_{0} \\
1-b_{1} & 1 & a_{1} \\
\vdots & \vdots & \vdots \\
1-b_{n-1} & n-1 & a_{n-1} \\
1-b_{n} & -\sum_{i=1}^{n}\left(1-b_{i}\right)-\sum_{i=0}^{n} a_{i} & a_{n}
\end{array}\right]
$$

Note that ${ }_{n+1} F_{n}$ includes the Gauss hypergeometric function as the case $n=1$.

In this article, we clarify a relationship between the system (1) and the function ${ }_{n+1} F_{n}$. For $n=1$ among them, the relationship between $P_{\mathrm{VI}}$ and the Gauss hypergeometric function is well known. Under the system (1) of the case $n=1$, we consider a specialization $p=\eta=0$. Then we obtain a Riccati equation

$$
t(t-1) \frac{d q}{d t}=\alpha_{1} q^{2}+\left\{\left(\alpha_{3}+\alpha_{0}\right) t-\left(\alpha_{0}+\alpha_{1}\right)\right\} q-\alpha_{3} t .
$$

Via a transformation of a dependent variable

$$
q=\frac{t(1-t)}{\alpha_{1}} \frac{d}{d t} \log \left\{(t-1)^{\alpha_{3}} x(t)\right\}
$$

we obtain the Gauss hypergeometric equation

$$
\left[\delta\left(\delta+\alpha_{2}+\alpha_{3}-1\right)-t\left(\delta+\alpha_{1}+\alpha_{2}+\alpha_{3}\right)\left(\delta+\alpha_{3}\right)\right] x=0 .
$$

The result of this article gives a natural extension of the above fact. For general $n$, we consider a specialization $p_{1}=\cdots=p_{n}=\eta=0$. Then we obtain the generalized hypergeometric equation by a certain transformation of dependent variables.

We also discuss a degeneration structure of the system (1) derived from the confluence of ${ }_{n+1} F_{n}$. The confluent hypergeometric functions ${ }_{n-r+1} F_{n}(r=1, \ldots, n+1)$ are defined by the power series

$$
{ }_{n-r+1} F_{n}\left[\begin{array}{c}
a_{r}, \ldots, a_{n} \\
b_{1}, \ldots, b_{n}
\end{array} ; t\right]=\sum_{i=0}^{\infty} \frac{\left(a_{r}\right)_{i} \cdots\left(a_{n}\right)_{i}}{\left(b_{1}\right)_{i} \cdots\left(b_{n}\right)_{i}} t^{i} .
$$


The process of confluence ${ }_{n-r+2} F_{n} \rightarrow{ }_{n-r+1} F_{n}$ is given by a replacement

$$
t \rightarrow \varepsilon t, \quad a_{r-1} \rightarrow \varepsilon^{-1},
$$

and taking a limit $\varepsilon \rightarrow 0$. We see that $x={ }_{n-r+1} F_{n}$ satisfy the confluent hypergeometric differential equations

$$
\left[\delta\left(\delta+b_{1}-1\right) \cdots\left(\delta+b_{n}-1\right)-t\left(\delta+a_{r}\right) \cdots\left(\delta+a_{n}\right)\right] x=0 .
$$

In this article, we propose a class of higher order Painlevé systems which admit particular solutions expressed in terms of ${ }_{n-r+1} F_{n}$.

Remark 1. In this article, we study a higher order generalization of $P_{\mathrm{VI}}$. On the other hand, for a multi-time generalization, it is known that the Garnier system admits a particular solution in terms of the Appell-Lauricella hypergeometric function $F_{D}$ [2].

This article is organized as follows. In Section 2, we derive a system of linear differential equations from the system (1) by a specialization $p_{1}=\cdots=p_{n}=\eta=0$. In Section 3, we give its fundamental solutions expressed in terms of the hypergeometric function ${ }_{n+1} F_{n}$ in a neighborhood of the singular point $t=0$. In Section 4 , we discuss a degeneration structure of the system (1) derived from the confluence of ${ }_{n+1} F_{n}$.

\section{Linear differential equations}

In this section, we derive a system of linear differential equations from the system (1) by a specialization $p_{1}=\cdots=p_{n}=\eta=0$.

We first consider a symmetric form of (1) in order to derive a system of linear differential equations. Let $x_{i}, y_{i}(i=0, \ldots, n)$ be dependent variables such that

$$
t(1-t) \frac{d}{d t} \log x_{n}=\sum_{i=1}^{n}\left\{\left(q_{i}-1\right)\left(q_{i}-t\right) p_{i}+\alpha_{2 i-1} q_{i}\right\}+t \alpha_{2 n+1}-(t+1) \eta,
$$

and

$$
x_{i-1}=\frac{x_{n} q_{i}}{t}, \quad y_{i-1}=\frac{t p_{i}}{x_{n}}, \quad i=1, \ldots, n, \quad y_{n}=-\frac{1}{x_{n}}\left(\sum_{j=1}^{n} q_{j} p_{j}+\eta\right) .
$$

Then we obtain a Hamiltonian system of $(2 n+2)$-th order

$$
\frac{d x_{i}}{d t}=\frac{\partial H}{\partial y_{i}}, \quad \frac{d y_{i}}{d t}=-\frac{\partial H}{\partial x_{i}}, \quad i=0, \ldots, n,
$$

with a Hamiltonian

$$
\begin{aligned}
H= & \frac{1}{t} \sum_{i=0}^{n}\left\{\frac{1}{2} x_{i}^{2} y_{i}^{2}-\alpha_{2 i+2}^{2 n-2 i-1} x_{i} y_{i}+\sum_{j=0}^{i-1} x_{i}\left(x_{i} y_{i}+\alpha_{2 i+1}\right) y_{j}\right\} \\
& +\frac{1}{1-t} \sum_{i=0}^{n} \sum_{j=0}^{n} x_{i}\left(x_{i} y_{i}+\alpha_{2 i+1}\right) y_{j},
\end{aligned}
$$

where

$$
\alpha_{k}^{l}= \begin{cases}0, & l \in \mathbb{Z}_{<0}, \\ \sum_{i=k}^{k+l} \alpha_{i}, & l \in \mathbb{Z}_{\geq 0} .\end{cases}
$$


The dependent variables $x_{i}, y_{i}$ and the fixed parameter $\eta$ satisfy a relation

$$
\sum_{i=0}^{n} x_{i} y_{i}+\eta=0
$$

Remark 2. The symmetric form (4) is suggested by the Hamiltonian system given in Theorem 3.2 of [4]. Their relationship is given by

$$
t=\frac{1}{t_{1}^{n+1}}, \quad x_{i}=\frac{w_{2 i+1}}{t_{1}^{i-n+\rho_{1}+\kappa_{2 n+1}-\kappa_{0}}}, \quad y_{i}=\frac{t_{1}^{i-n+\rho_{1}+\kappa_{2 n+1}-\kappa_{0}} \varphi_{2 i+1}}{n+1},
$$

and

$$
\begin{aligned}
& \eta=\sum_{j=0}^{n} \frac{\rho_{1}+\kappa_{2 i}-\kappa_{2 i+1}}{n+1}, \quad \alpha_{2 i}=\frac{1+\kappa_{2 i-1}-2 \kappa_{2 i}+\kappa_{2 i+1}}{n+1}, \\
& \alpha_{2 i+1}=\frac{\kappa_{2 i}-2 \kappa_{2 i+1}+\kappa_{2 i+2}}{n+1},
\end{aligned}
$$

for $i=0, \ldots, n$.

Remark 3. The system (4), or equivalently the system (1), admits the affine Weyl group symmetry of type $A_{2 n+1}^{(1)}$; see Appendix B.

We can derive easily a system of linear differential equations from the symmetric form by the specialization $y_{0}=\cdots=y_{n}=\eta=0$, which is equivalent to $p_{1}=\cdots=p_{n}=\eta=0$. Let $E_{i, j}$ be the matrix unit defined by

$$
E_{i, j}=\left(\delta_{i, k} \delta_{j, l}\right)_{k, l=0}^{n} .
$$

For example, in the case $n=2$, it is explicitly given as

$$
E_{1,1}=\left[\begin{array}{ll}
1 & 0 \\
0 & 0
\end{array}\right], \quad E_{1,2}=\left[\begin{array}{ll}
0 & 1 \\
0 & 0
\end{array}\right], \quad E_{2,1}=\left[\begin{array}{ll}
0 & 0 \\
1 & 0
\end{array}\right], \quad E_{2,2}=\left[\begin{array}{ll}
0 & 0 \\
0 & 1
\end{array}\right] .
$$

Then we obtain

Proposition 1. The system (4) admits a specialization

$$
y_{i}=0, \quad i=0, \ldots, n, \quad \eta=0 .
$$

Then a vector of the variables $\mathbf{x}={ }^{t}\left(x_{0}, \ldots, x_{n}\right)$ satisfies a system of linear differential equations on $\mathbb{P}^{1}(\mathbb{C})$

$$
\frac{d \mathbf{x}}{d t}=\left(\frac{A_{0}}{t}+\frac{A_{1}}{1-t}\right) \mathbf{x}
$$

with

$$
A_{0}=\sum_{i=0}^{n-1}\left(-\alpha_{2 i+2}^{2 n-2 i-1}\right) E_{i, i}+\sum_{i=0}^{n-1} \sum_{j=i+1}^{n} \alpha_{2 j+1} E_{i, j}, \quad A_{1}=\sum_{i=0}^{n} \sum_{j=0}^{n} \alpha_{2 j+1} E_{i, j} .
$$

Furthermore, the system (5) is of Fuchsian type with regular singular points at $t=0,1, \infty$. The data of eigenvalues of its residue matrices is given as

$$
\begin{aligned}
& -\alpha_{2}^{2 n-1}, \ldots,-\alpha_{2 n}^{1}, 0 \quad \text { at } t=0, \\
& 0, \ldots, 0,-\sum_{i=0}^{n} \alpha_{2 i+1} \quad \text { at } t=1, \\
& \alpha_{1}^{2 n}, \ldots, \alpha_{2 n-1}^{2}, \alpha_{2 n+1} \quad \text { at } t=\infty .
\end{aligned}
$$


Remark 4. The system (4) also admits a specialization

$$
x_{i}=0, \quad i=0, \ldots, n-1, \quad x_{n} y_{n}+\eta=0, \quad \eta-\alpha_{2 n+1}=0,
$$

which is equivalent to $q_{1}=\cdots=q_{n}=\eta-\alpha_{2 n+1}=0$. Then a vector of the variables $\mathbf{y}=$ ${ }^{t}\left(y_{0}, \ldots, y_{n}\right)$ satisfies a system of linear differential equations

$$
\frac{d \mathbf{y}}{d t}=\left(\frac{A_{0}}{t}+\frac{A_{1}}{1-t}\right) \mathbf{y},
$$

with

$$
\begin{aligned}
& A_{0}=\sum_{i=0}^{n-1} \alpha_{2 i+2}^{2 n-2 i-1} E_{i, i}+\sum_{i=1}^{n-1} \sum_{j=0}^{i-1}\left(-\alpha_{2 i+1}\right) E_{i, j}+\sum_{j=0}^{n} \alpha_{2 n+1} E_{n, j}, \\
& A_{1}=\sum_{i=0}^{n-1} \sum_{j=0}^{n}\left(-\alpha_{2 i+1}\right) E_{i, j}+\sum_{j=0}^{n} \alpha_{2 n+1} E_{n, j} .
\end{aligned}
$$

We always assume that

$$
\alpha_{2 i}^{2 j-1} \notin \mathbb{Z}, \quad \sum_{i=0}^{n} \alpha_{2 i+1} \notin \mathbb{Z}, \quad \alpha_{2 i-1}^{2 j-1} \notin \mathbb{Z}, \quad i=1, \ldots, n, \quad j=1, \ldots, n-i+1 .
$$

In the next section, we describe fundamental solutions of the system (5) in a neighborhood of the singular point $t=0$ explicitly.

\section{Hypergeometric function ${ }_{n+1} F_{n}$}

In this section, we give fundamental solutions of the system (5) expressed in terms of the hypergeometric function ${ }_{n+1} F_{n}$ in a neighborhood of the singular point $t=0$.

For each $k=0, \ldots, n$, we consider a gauge transformation

$$
\mathbf{x}^{k}=t^{\alpha_{2 k+2}^{2 n-2 k-1}}\left(\sum_{i=0}^{n-k-1} t^{-1} E_{i, i+k+1}+\sum_{i=n-k}^{n} E_{i, i-n+k}\right) \mathbf{x} .
$$

Then the system (5) is transformed into

$$
\frac{d \mathbf{x}^{k}}{d t}=\left(\frac{A_{0}^{k}}{t}+\frac{A_{1}^{k}}{1-t}\right) \mathbf{x}^{k}
$$

with

$$
\begin{aligned}
A_{0}^{k} & =\sum_{i=0}^{n-1}\left(-\alpha_{2 k+2 i+4}^{2 n-2 i-1}\right) E_{i, i}+\sum_{i=0}^{n-1} \sum_{j=i+1}^{n} \alpha_{2 j+2 k+3} E_{i, j}, \\
A_{1}^{k} & =\sum_{i=0}^{n} \sum_{j=0}^{n} \alpha_{2 j+2 k+3} E_{i, j} .
\end{aligned}
$$

Recall that indices of the fixed parameters $\alpha_{i}$ are congruent modulo $2 n+2$, from which we have $\alpha_{2 k+2 n+2}^{1}=\alpha_{2 k}^{1}$. We also consider a formal power series of $\mathbf{x}^{k}$ at $t=0$

$$
\mathbf{x}^{k}=\sum_{i=0}^{\infty} \mathbf{x}_{i}^{k} t^{i}
$$


Then the system (6) implies

$$
\begin{aligned}
& A_{0}^{k} \mathbf{x}_{0}^{k}=\mathbf{0}, \\
& \left\{A_{0}^{k}-(i+1) I\right\} \mathbf{x}_{i+1}^{k}=\left(A_{0}^{k}-A_{1}^{k}-i I\right) \mathbf{x}_{i}^{k}, \quad i \in \mathbb{Z}_{\geq 0},
\end{aligned}
$$

where $I$ stands for the identity matrix. The matrices $A_{0}^{k}$ and $A_{0}^{k}-(i+1) I$ are of rank $n$ and $n+1$, respectively. It follows that the recurrence formula (7) admits one parameter family of solutions.

For each $k=0, \ldots, n$, we can show that a sequence of vectors

$$
\mathbf{x}_{i}^{k}=\left[\begin{array}{c}
\prod_{j=0}^{n-1} \frac{\left(\alpha_{2 k-2 j+1}^{2 j}\right)_{i+1}}{\left(\alpha_{2 k-2 j}^{2 j+1}\right)_{i+1}} \cdot \frac{\left(\alpha_{2 k+3}^{2 n}\right)_{i}}{\left(\alpha_{2 k+2}^{2 n+1}\right)_{i}} \\
\prod_{j=0}^{n-2} \frac{\left(\alpha_{2 k-2 j+1}^{2 j}\right)_{i+1}}{\left(\alpha_{2 k-2 j}^{2 j+1}\right)_{i+1}} \cdot \frac{\left(\alpha_{2 k+5}^{2 n-2}\right)_{i}\left(\alpha_{2 k+3}^{2 n}\right)_{i}}{\left(\alpha_{2 k+4}^{2 n-1}\right)_{i}\left(\alpha_{2 k+2}^{2 n+1}\right)_{i}} \\
\vdots \\
\frac{\left(\alpha_{2 k+1}\right)_{i+1}}{\left(\alpha_{2 k}^{1}\right)_{i+1}} \prod_{j=0}^{n-1} \frac{\left(\alpha_{2 k+2 j+3}^{2 n-2 j}\right)_{i}}{\left(\alpha_{2 k+2 j+2}^{2 n-2 j+1}\right)_{i}} \\
\prod_{j=0}^{n} \frac{\left(\alpha_{2 k+2 j+3}^{2 n-2 j}\right)_{i}}{\left(\alpha_{2 k+2 j+2}^{2 n-2 j+1}\right)_{i}}
\end{array}\right], \quad i \in \mathbb{Z}_{\geq 0},
$$

satisfies the recurrence formula (7) by a direct computation. Note that $\alpha_{2 k+2}^{2 n+1}=1$. Therefore we arrive at

Theorem 1. On a domain $|t|<1$, the system (5) admits fundamental solutions

$$
\mathbf{x}=t^{-\alpha_{2 k+2}^{2 n-2 k-1}}\left[\begin{array}{c}
f^{k, k} \\
\vdots \\
f^{k, 0} \\
t f^{k, n} \\
\vdots \\
t f^{k, k+1}
\end{array}\right], \quad k=0, \ldots, n
$$

where

$$
f^{k, l}=\prod_{i=1}^{l} \frac{\alpha_{2 k-2 i+3}^{2 i-2}}{\alpha_{2 k-2 i+2}^{2 i-1}} \cdot{ }_{n+1} F_{n}\left[\begin{array}{c}
a_{0}, \ldots, a_{n} \\
b_{1}, \ldots, b_{n}
\end{array} ; t\right],
$$

with

$$
\begin{array}{lll}
a_{0}=\alpha_{2 k-2 n+1}^{2 n}, & & \\
a_{i}=1+\alpha_{2 k-2 i+3}^{2 i-2}, & b_{i}=1+\alpha_{2 k-2 i+2}^{2 i-1}, & i=1, \ldots, l, \\
a_{i}=\alpha_{2 k-2 i+3}^{2 i-2}, & b_{i}=\alpha_{2 k-2 i+2}^{2 i-1}, & i=l+1, \ldots, n .
\end{array}
$$

Corollary 1. If the vector $\mathbf{x}={ }^{t}\left(x_{0}, \ldots, x_{n}\right)$ satisfies the system (5), each component $x_{i}$ satisfies the generalized hypergeometric equation (2) with

$$
\begin{array}{lll}
a_{0}=\alpha_{1}^{2 n}, & & \\
a_{j}=1+\alpha_{2 n-2 j+3}^{2 j-2}, & b_{j}=1+\alpha_{2 n-2 j+2}^{2 j-1}, & j=1, \ldots, n-i, \\
a_{j}=\alpha_{2 n-2 j+3}^{2 j-2}, & b_{j}=\alpha_{2 n-2 j+2}^{2 j-1}, & j=n-i+1, \ldots, n .
\end{array}
$$

Remark 5. The system (5) have been already studied by Okubo-Takano-Yoshida [3]. They considered the Fuchsian differential equation of Okubo type and obtained its fundamental solutions at singular points $t=0,1$. 


\section{Degeneration structure}

In this section, we discuss a degeneration structure of the system (1) derived from the confluence of ${ }_{n+1} F_{n}$.

For each $r=1, \ldots, n+1$, we consider a Hamiltonian system

$$
{ }_{n-r+1} \mathcal{H}_{n}: \quad \frac{d x_{i}}{d t}=\frac{\partial H}{\partial y_{i}}, \quad \frac{d y_{i}}{d t}=-\frac{\partial H}{\partial x_{i}}, \quad i=0, \ldots, n,
$$

with a Hamiltonian

$$
t H=\sum_{i=0}^{n} \frac{1}{2} x_{i} y_{i}\left(x_{i} y_{i}-2 \alpha_{2 i+2}^{2 n-2 i-1}\right)+\sum_{i=0}^{r-2} x_{i+1} y_{i}+\sum_{i=r-1}^{n}\left\{t x_{0}+\sum_{j=i+1}^{n} x_{j}\left(x_{j} y_{j}+\alpha_{2 j+1}\right)\right\} y_{i} .
$$

Here $\alpha_{i}(i=0, \ldots, 2 n+1)$ and $\eta$ are fixed parameters satisfying

$$
\alpha_{2 i}=0, \quad i=0, \ldots, r-1, \quad \sum_{j=0}^{n} \alpha_{2 j+1}+\sum_{j=r}^{n} \alpha_{2 j}=1,
$$

and

$$
\sum_{j=0}^{n} x_{j} y_{j}+\eta=0
$$

Note that

$$
\alpha_{2 i+2}^{2 n-2 i-1}=\sum_{j=2 i+2}^{2 n+1} \alpha_{j}=\sum_{j=i+1}^{n} \alpha_{2 j+1}+\sum_{j=\max (r, i+1)}^{n} \alpha_{2 j}
$$

The system ${ }_{n-r+1} \mathcal{H}_{n}$ is obtained from ${ }_{n-r+2} \mathcal{H}_{n}$ by a replacement

$$
\begin{aligned}
t \rightarrow \varepsilon t, \quad \alpha_{2 r-2} & \rightarrow-\varepsilon^{-1}, \quad \alpha_{2 r-1} \rightarrow \alpha_{2 r-1}+\varepsilon^{-1}, \\
x_{i} & \rightarrow \varepsilon^{-1} x_{i}, \quad y_{i} \rightarrow \varepsilon y_{i}, \quad i=0, \ldots, r-2 .
\end{aligned}
$$

and taking a limit $\varepsilon \rightarrow 0$, where ${ }_{n+1} \mathcal{H}_{n}$ stands for the system (4).

Remark 6. Such degenerate systems also can be rewritten into the Hamiltonian systems in terms of the canonical coordinates. We give their explicit formulas for $n=1$ and $n=2$ in Appendix A.

The system ${ }_{n-r+1} \mathcal{H}_{n}$ admits a specialization

$$
y_{i}=0, \quad i=0, \ldots, n, \quad \eta=0 .
$$

Then a vector of the variables $\mathbf{x}={ }^{t}\left(x_{0}, \ldots, x_{n}\right)$ satisfies a system of linear differential equations

$$
{ }_{n-r+1} \mathcal{L}_{n}: \frac{d \mathbf{x}}{d t}=\left(\frac{A_{0}}{t}+A_{1}\right) \mathbf{x},
$$

with

$$
\begin{aligned}
& A_{0}=\sum_{i=0}^{n-1}\left(-\alpha_{2 i+2}^{2 n-2 i-1}\right) E_{i, i}+\sum_{i=0}^{r-2} E_{i, i+1}+\sum_{i=r-1}^{n-1} \sum_{j=i+1}^{n} \alpha_{2 j+1} E_{i, j}, \\
& A_{1}=\sum_{i=r-1}^{n} E_{i, 0} .
\end{aligned}
$$

Note that ${ }_{n-r+1} \mathcal{L}_{n}$ is obtained from ${ }_{n-r+2} \mathcal{L}_{n}$ through the above process of confluence.

In a similar manner as Section 3, we arrive at 
Theorem 2. On a domain $|t|<1$, the system ${ }_{n-r+1} \mathcal{L}_{n}$ admits fundamental solutions

$$
\mathbf{x}=t^{-\alpha_{2 k+2}^{2 n-2 k-1}}\left[\begin{array}{c}
f_{r}^{k, k} \\
\vdots \\
f_{r}^{k, 0} \\
t f_{r}^{k, n} \\
\vdots \\
t f_{r}^{k, k+1}
\end{array}\right], \quad k=0, \ldots, n
$$

where

$$
f_{r}^{k, l}=\prod_{\substack{1 \leq i \leq l \\
\bmod [k-i+1, n+1] \geq r}} \alpha_{2 k-2 i+3}^{2 i-2} \prod_{1 \leq i \leq l} \frac{1}{\alpha_{2 k-2 i+2}^{2 i-1}} \cdot{ }_{n-r+1} F_{n}\left[\begin{array}{c}
a_{r}, \ldots, a_{n} \\
b_{1}, \ldots, b_{n}
\end{array} ; t\right],
$$

and

$$
\bmod [i, n+1]=i-m(n+1) \quad \text { for } \quad m(n+1) \leq i<(m+1)(n+1)
$$

Here the parameters $a_{r}, \ldots, a_{n}$ are given by

$$
a_{i}=\alpha_{2 r-2 i-1}^{2 k-2 r+2 i+2}, \quad i=r, \ldots, n,
$$

for $k+1 \leq r$ and $l<k+2$;

$$
\begin{array}{ll}
a_{i}=1+\alpha_{2 r-2 i-1}^{2 k-2 r+2 i+2}, & i=r, \ldots, r-k+l-2, \\
a_{i}=\alpha_{2 r-2 i-1}^{2 k-2 r+2 i+2}, & i=r-k+l-1, \ldots, n,
\end{array}
$$

for $k+1 \leq r$ and $k+2 \leq l$;

$$
\begin{aligned}
a_{i} & =\alpha_{2 r-2 i-1}^{2 k-2 r+2 i+2}, & i & =r, \ldots, n+r-k-1, \\
a_{i} & =1+\alpha_{2 r-2 i-1}^{2 k-2 r+2 i+2}, & & i=n+r-k, \ldots, n+r-k+l-1, \\
a_{i} & =\alpha_{2 r-2 i-1}^{2 k-2 r+2 i+2}, & i & =n+r-k+l, \ldots, n,
\end{aligned}
$$

for $r<k+1$ and $l<k-r+1$;

$$
\begin{array}{ll}
a_{i}=\alpha_{2 r-2 i-1}^{2 k-2 r+2 i+2}, & i=r, \ldots, n+r-k-1, \\
a_{i}=1+\alpha_{2 r-2 i-1}^{2 k-2 r+2 i+2}, & i=n+r-k, \ldots, n,
\end{array}
$$

for $r<k+1$ and $k-r+1 \leq l<k+2$;

$$
\begin{array}{ll}
a_{i}=1+\alpha_{2 r-2 i-1}^{2 k-2 i+2}, & i=r, \ldots, r-k+l-2, \\
a_{i}=\alpha_{2 r-2 i-1}^{2 k-2 r+2 i+2}, & i=r-k+l-1, \ldots, n+r-k-1, \\
a_{i}=1+\alpha_{2 r-2 i-1}^{2 k-2 r+2 i+2}, & i=n+r-k, \ldots, n,
\end{array}
$$

for $r<k+1$ and $k+2 \leq l$. The parameters $b_{1}, \ldots, b_{n}$ are given by

$$
\begin{aligned}
b_{i} & =1+\alpha_{2 k-2 i+2}^{2 i-1}, & i & =1, \ldots, l, \\
b_{i} & =\alpha_{2 k-2 i+2}^{2 i-1}, & i & =l+1, \ldots, n .
\end{aligned}
$$


Corollary 2. If the vector $\mathbf{x}={ }^{t}\left(x_{0}, \ldots, x_{n}\right)$ satisfies the system ${ }_{n-r+1} \mathcal{L}_{n}$, each component $x_{i}$ satisfies the confluent hypergeometric equation (3) with

$$
a_{j}=1+\alpha_{2 r-2 j-1}^{2 n-2 r+2 j+2}, \quad j=r, \ldots, n,
$$

for $i \leq r-1$,

$$
\begin{array}{ll}
a_{j}=1+\alpha_{2 r-2 j-1}^{2 n-2 r+2 j+2}, & j=r, \ldots, n+r-i-1, \\
a_{j}=\alpha_{2 r-2 j-1}^{2 n-2 r+2 j+2}, & j=n+r-i, \ldots, n,
\end{array}
$$

for $r-1<i$ and

$$
\begin{array}{ll}
b_{j}=1+\alpha_{2 n-2 j+2}^{2 j-1}, & j=1, \ldots, n-i, \\
b_{j}=\alpha_{2 n-2 j+2}^{2 j-1}, & j=n-i+1, \ldots, n,
\end{array}
$$

for any $i$.

\section{A Canonical Hamiltonian system}

The systems ${ }_{n-r+1} \mathcal{H}_{n}$ can be rewritten into the Hamiltonian systems in terms of canonical coordinates. In this section, we give their explicit formulas for $n=1$ and $n=2$. Note that ${ }_{3-r} \mathcal{H}_{2}$ appear in the classification of the fourth order Painlevé type differential equations [5].

\section{A.1 Case $n=1, r=1$}

Under the system ${ }_{1} \mathcal{H}_{1}$, we take canonical coordinates

$$
q=\frac{x_{0}}{x_{1}}, \quad p=-\frac{x_{1}\left(x_{1} y_{1}+\alpha_{3}\right)}{x_{0}} .
$$

Via a transformation of the independent variable $t \rightarrow-t$, we obtain a Hamiltonian system

$$
\frac{d q}{d t}=\frac{\partial H}{\partial p}, \quad \frac{d p}{d t}=-\frac{\partial H}{\partial q},
$$

with a Hamiltonian

$$
t H=q(q-1) p(p+t)-q p\left(\eta+\alpha_{2}-\alpha_{3}\right)+\left(\eta-\alpha_{3}\right) p+t \alpha_{3} q .
$$

It is equivalent to the fifth Painlevé equation.

\section{A.2 Case $n=1, r=2$}

Under the system ${ }_{0} \mathcal{H}_{1}$, we take canonical coordinates

$$
q=\frac{x_{1}}{x_{0}}, \quad p=x_{0} y_{1} .
$$

Then we obtain a Hamiltonian system

$$
\frac{d q}{d t}=\frac{\partial H}{\partial p}, \quad \frac{d p}{d t}=-\frac{\partial H}{\partial q},
$$

with a Hamiltonian

$$
t H=q^{2} p(p-1)+\left(\eta+\alpha_{3}\right) q p+t p-\eta q .
$$

It is equivalent to the third Painlevé equation. 


\section{A.3 Case $n=2, r=1$}

Under the system ${ }_{2} \mathcal{H}_{2}$, we take canonical coordinates

$$
q_{1}=\frac{x_{0}}{x_{1}}, \quad p_{1}=-\frac{x_{1}\left(x_{1} y_{1}+\alpha_{3}\right)}{x_{0}}, \quad q_{2}=\frac{x_{0}}{x_{2}}, \quad p_{2}=-\frac{x_{2}\left(x_{2} y_{2}+\alpha_{5}\right)}{x_{0}} .
$$

Via a transformation of the independent variable $t \rightarrow-t$, we obtain a Hamiltonian system

$$
\frac{d q_{i}}{d t}=\frac{\partial H}{\partial p_{i}}, \quad \frac{d p_{i}}{d t}=-\frac{\partial H}{\partial q_{i}}, \quad i=1,2,
$$

with a Hamiltonian

$$
\begin{aligned}
t H= & q_{1}\left(q_{1}-1\right) p_{1}\left(p_{1}+t\right)-\left(\eta+\alpha_{2}-\alpha_{3}-\alpha_{5}\right) q_{1} p_{1}+\left(\eta-\alpha_{3}-\alpha_{5}\right) p_{1} \\
& +\alpha_{3} t q_{1}+\left(q_{1}-1\right) p_{1} q_{2} p_{2}+\left(q_{1}-1\right)\left(q_{1} p_{1}+\alpha_{3}\right) p_{2} \\
& +q_{2}\left(q_{2}-1\right) p_{2}\left(p_{2}+t\right)-\left(\eta+\alpha_{2}+\alpha_{4}-\alpha_{5}\right) q_{2} p_{2}+\left(\eta-\alpha_{5}\right) p_{2}+\alpha_{5} t q_{2} .
\end{aligned}
$$

\section{A.4 Case $n=2, r=2$}

Under the system ${ }_{1} \mathcal{H}_{2}$, we take canonical coordinates

$$
q_{1}=-\frac{x_{1}}{x_{0}}, \quad p_{1}=1-x_{0} y_{1}, \quad q_{2}=-\frac{x_{2}}{x_{0}}, \quad p_{2}=-x_{0} y_{2} .
$$

Via a transformation of the independent variable $t \rightarrow-t$, we obtain a Hamiltonian system

$$
\frac{d q_{i}}{d t}=\frac{\partial H}{\partial p_{i}}, \quad \frac{d p_{i}}{d t}=-\frac{\partial H}{\partial q_{i}}, \quad i=1,2,
$$

with a Hamiltonian

$$
\begin{aligned}
t H= & q_{1}^{2} p_{1}\left(p_{1}-1\right)+\left(\eta+\alpha_{3}\right) q_{1} p_{1}+t p_{1}-\alpha_{3} q_{1}+q_{1} p_{1} q_{2} p_{2}+p_{1} q_{2}\left(q_{2} p_{2}+\alpha_{5}\right) \\
& +q_{2}^{2} p_{2}\left(p_{2}-1\right)+\left(\eta+\alpha_{3}+\alpha_{4}+\alpha_{5}\right) q_{2} p_{2}+t p_{2}-\alpha_{5} q_{2} .
\end{aligned}
$$

\section{A.5 Case $n=2, r=3$}

Under the system ${ }_{0} \mathcal{H}_{2}$, we take canonical coordinates

$$
q_{1}=-\frac{x_{1}}{x_{0}}, \quad p_{1}=1-x_{0} y_{1}, \quad q_{2}=-\frac{x_{2}}{x_{0}}, \quad p_{2}=-x_{0} y_{2} .
$$

Via a transformation of the independent variable $t \rightarrow-t$, we obtain a Hamiltonian system

$$
\frac{d q_{i}}{d t}=\frac{\partial H}{\partial p_{i}}, \quad \frac{d p_{i}}{d t}=-\frac{\partial H}{\partial q_{i}}, \quad i=1,2,
$$

with a Hamiltonian

$$
\begin{aligned}
t H= & q_{1}^{2} p_{1}\left(p_{1}-1\right)+\left(\eta+\alpha_{3}\right) q_{1} p_{1}-\alpha_{3} q_{1}+q_{1} p_{1} q_{2} p_{2}+p_{1} q_{2} \\
& +q_{2}^{2} p_{2}^{2}+\left(\eta+\alpha_{3}+\alpha_{5}\right) q_{2} p_{2}+t p_{2}-q_{2} .
\end{aligned}
$$




\section{B Affine Weyl group symmetry}

The system (4) admits the affine Weyl group symmetry of type $A_{2 n+1}^{(1)}$. In this section, we describe its action on the dependent variables and parameters.

Recall that the affine Weyl group of type $A_{2 n+1}^{(1)}$ is generated by the transformations $r_{i}$ $(i=0, \ldots, 2 n+1)$ with the fundamental relations

$$
\begin{array}{ll}
r_{i}^{2}=1, & i=0, \ldots, 2 n+1, \\
\left(r_{i} r_{j}\right)^{2-a_{i, j}}=0, & i, j=0, \ldots, 2 n+1, \quad i \neq j,
\end{array}
$$

where

$$
\begin{array}{ll}
a_{i, i}=2, & i=0, \ldots, 2 n+1, \\
a_{i, i+1}=a_{2 n+1,0}=a_{i+1, i}=a_{0,2 n+1}=-1, & i=0, \ldots, 2 n, \\
a_{i, j}=0, & \text { otherwise } .
\end{array}
$$

We define the Poisson structure by

$$
\left\{x_{i}, y_{j}\right\}=-\delta_{i, j}, \quad i, j=0, \ldots, n .
$$

Then the Hamiltonian system (4) is invariant under the birational transformations $r_{0}, \ldots, r_{2 n+1}$ defined by

$$
\begin{aligned}
& r_{0}\left(x_{j}\right)=t^{-\alpha_{0}} x_{j}, \quad r_{0}\left(y_{j}\right)=t^{\alpha_{0}}\left(y_{j}+\frac{\alpha_{0}}{x_{n}-t x_{0}}\left\{x_{n}-t x_{0}, y_{j}\right\}\right), \\
& r_{2 i+1}\left(x_{j}\right)=x_{j}+\frac{\alpha_{2 i+1}}{y_{i}}\left\{y_{i}, x_{j}\right\}, \quad r_{2 i+1}\left(y_{j}\right)=y_{j}, \quad i=0, \ldots, n-1, \\
& r_{2 i}\left(x_{j}\right)=x_{j}, \quad r_{2 i}\left(y_{j}\right)=y_{j}+\frac{\alpha_{2 i}}{x_{i-1}-x_{i}}\left\{x_{i-1}-x_{i}, y_{j}\right\}, \quad i=1, \ldots, n, \\
& r_{2 n+1}\left(x_{j}\right)=t^{\alpha_{2 n+1}}\left(x_{j}+\frac{\alpha_{2 n+1}}{y_{n}}\left\{y_{n}, x_{j}\right\}\right), \quad r_{2 n+1}\left(y_{j}\right)=t^{-\alpha_{2 n+1}} y_{j},
\end{aligned}
$$

for $j=0, \ldots, n$ and

$$
r_{i}\left(\alpha_{j}\right)=\alpha_{j}-a_{i, j} \alpha_{i}, \quad r_{i}(\eta)=\eta+(-1)^{i} \alpha_{i}, \quad i, j=0, \ldots, 2 n+1 .
$$

The group of symmetries $\left\langle r_{0}, \ldots, r_{2 n+1}\right\rangle$ is isomorphic to the affine Weyl group of type $A_{2 n+1}^{(1)}$.

\section{Acknowledgements}

The author would like to express his gratitude to Mr. Masaomi Miyamoto of Kobe University for fruitful discussion. The author is also grateful to Professors Masatoshi Noumi, Hidetaka Sakai, Teruhisa Tsuda and Yasuhiko Yamada for helpful comments and advices.

\section{References}

[1] Fuji K., Suzuki T., Drinfeld-Sokolov hierarchies of type $A$ and fourth order Painlevé systems, Funkcial. Ekvac. 53 (2010), 143-167, arXiv:0904.3434.

[2] Iwasaki K., Kimura H., Shimomura S., Yoshida M., From Gauss to Painlevé. A modern theory of special functions, Aspects of Mathematics, Vol. E16, Friedr. Vieweg \& Sohn, Braunschweig, 1991.

[3] Okubo K., Takano K., Yoshida S., A connection problem for the generalized hypergeometric equation, Funkcial. Ekvac. 31 (1988), 483-495.

[4] Suzuki T., A class of higher order Painlevé systems arising from integrable hierarchies of type $A$, arXiv:1002.2685.

[5] Sakai H., Private communication.

[6] Tsuda T., UC hierarchy and monodromy preserving deformation, arXiv:1007.3450. 